BMJ Open

Diabetes

Research

\& Care

\section{Metformin in pregnancy and risk of adverse long-term outcomes: a register- based cohort study}

To cite: Brand KMG, Saarelainen L, Sonajalg J, et al. Metformin in pregnancy and risk of adverse long-term outcomes: a register-based cohort study. BMJ Open Diab Res Care 2022;10:e002363. doi:10.1136/

bmjdrc-2021-002363

- Additional supplemental material is published online only. To view, please visit the journal online (http://dx.doi. org/10.1136/bmjdrc-2021002363).

Received 9 August 2021 Accepted 11 December 2021

Check for updates

(c) Author(s) (or their employer(s)) 2022. Re-use permitted under CC BY-NC. No commercial re-use. See rights and permissions. Published by BMJ.

${ }^{1}$ Merck KGaA, Darmstadt, Germany

${ }^{2}$ Global Database Studies, IQVIA, Espoo, Finland

${ }^{3}$ Global Database Studies, IQVIA, Tartu, Estonia ${ }^{4}$ PEDEGO Research Unit, Medical Research Centre Oulu, Oulu University Hospital, Oulu, Finland

${ }^{5}$ University of Oulu, Oulu, Finland

${ }^{6}$ Global Database Studies, IQVIA, Solna, Sweden

Correspondence to Dr Kerstin M G Brand; kerstin.brand@merckgroup. com

\section{ABSTRACT}

Introduction This study aimed to investigate if maternal pregnancy exposure to metformin is associated with increased risk of long-term and short-term adverse outcomes in the child.

Research design and methods This register-based cohort study from Finland included singleton children born 2004-2016 with maternal pregnancy exposure to metformin or insulin (excluding maternal type 1 diabetes): metformin only ( $n=3967)$, insulin only $(n=5273)$ and combination treatment (metformin and insulin; $n=889$ ). The primary outcomes were long-term offspring obesity, hypoglycemia, hyperglycemia, diabetes, hypertension, polycystic ovary syndrome, and challenges in motorsocial development. In a sensitivity analysis, the primary outcomes were investigated only among children with maternal gestational diabetes. Secondary outcomes were adverse outcomes at birth. Analyses were conducted using inverse- probability of treatment weighting (IPTW), with insulin as reference.

Results Exposure to metformin or combination treatment versus insulin was not associated with increased risk of long-term outcomes in the main or sensitivity analyses. Among the secondary outcomes, increased risk of small for gestational age (SGA) was observed for metformin (IPTWweighted OR 1.65, 95\% Cl 1.16 to 2.34); increased risk of large for gestational age, preterm birth and hypoglycemia was observed for combination treatment. No increased risk was observed for neonatal mortality, hyperglycemia, or major congenital anomalies.

Conclusions This study found no increased long-term risk associated with pregnancy exposure to metformin (alone or in combination with insulin), compared with insulin. The increased risk of SGA associated with metformin versus insulin suggests caution in pregnancies with at-risk fetal undernutrition. The increased risks of adverse outcomes at birth associated with combination treatment may reflect confounding by indication or severity.

\section{INTRODUCTION}

Metformin is the first-line monotherapy for type 2 diabetes mellitus (T2DM) after failure of lifestyle modifications in all major guidelines and the most commonly prescribed drug for T2DM worldwide. ${ }^{12}$ While no approved indication in

\section{Significance of this study}

What is already known about this subject?

> The effects of pregnancy exposure to metformin on child outcomes are limited. The association between metformin exposure in pregnancy and adverse child outcomes, notably adverse weight outcomes at birth and long term is limited. The link of maternal drug exposure to the outcomes to mother and child at birth have been rarely assessed on a whole country level. Being a safety study, the risk for adverse outcomes with metformin was compared with the most commonly used medication for gestational hyperglycemia, insulin. Protective effects are therefore not highlighted.

What are the new findings?

- Maternal exposure to metformin and combination treatment of metformin and insulin was not associated with long-term increased risk of obesity, hypoglycemia, hyperglycemia, diabetes, or challenges in motor-social development, compared with insulin.

- The results were generally consistent in sensitivity analyses restricted to children with maternal gestational diabetes.

- The analyses of adverse outcomes at birth showed significantly increased risk of being small for gestational age associated with exposure to metformin, compared with insulin. It remains to be determined whether this is relative to an overall increase in body weight due to insulin.

- Combination treatment of metformin and insulin was associated with increased risk of being large for gestational age, preterm birth and hypoglycemia, although this association may be explained by confounding, namely more severe maternal hyperglycemia.

How might these results change the focus of research or clinical practice?

- This study represents the largest data set of pregnancies exposed with metformin to date, especially regarding long-term outcomes. It adds to the emerging positive benefit-risk balance of the use of metformin during pregnancy. 
pregnant women exists, metformin is increasingly being used for the treatment of gestational diabetes (GDM). ${ }^{3}$ In women with polycystic ovary syndrome (PCOS), metformin is commonly prescribed off-label to improve anovulation and conception, with some evidence also suggesting decreased risk of adverse pregnancy outcomes, if metformin is continued into pregnancy. ${ }^{4}$

Although generally considered safe in pregnancy, metformin, unlike insulin, ${ }^{5-7}$ crosses the placenta and may have comparable plasma concentrations in the mother and child at birth. ${ }^{8}$ Several studies have investigated the association between metformin and fetal outcomes at birth; while diverse in analytic approach and study population, results have indicated that metformin may be associated with lower risk of being large for gestational age (LGA) and neonatal hypoglycemia, compared with insulin. ${ }^{9}$ Although increased risk of preterm birth was observed relative to insulin in one study, ${ }^{10}$ the majority of previous data have suggested no association. ${ }^{9}$ No association has been observed for the risk of major congenital anomalies (MCAs). ${ }^{11-13}$

Current evidence on long-term outcomes of maternal metformin in the child mainly consists of follow-up studies of randomized controlled trials (RCTs) in GDM, ${ }^{10}{ }^{14}$ which did not differentiate metformin use alone or in combination with insulin in comparison with insulin. In these, metformin use was sometimes associated with higher offspring weight or weight-height ratio between 1 and 9 years of age. ${ }^{15-17}$ In a large observational study, no difference was observed at 4 years. ${ }^{18}$ Pregnancy exposure with metformin versus placebo for PCOS from first trimester to delivery ${ }^{19}$ was associated with higher child weight or body mass index (BMI) z-score at the age of 4-10 years, ${ }^{20}{ }^{21}$ whereas metformin versus placebo for pregnancy obesity did not lead to a difference in weight in 4 -year-old children. ${ }^{22}$ Evidence regarding long-term neurologic development has indicated no increased risk. ${ }^{15} 18202123$

There is an important need to further elucidate the long-term effects of in utero exposure to metformin. While the short-term safety of metformin during pregnancy has been investigated in several large studies, ${ }^{10}{ }^{24}$ the primary objective of this study was to investigate whether maternal pregnancy exposure to metformin is associated with long-term adverse outcomes in the child, including obesity, hypoglycemia, hyperglycemia, hypertension, diabetes, PCOS and challenges in motor-social development (MSD). The secondary objective was to investigate potential adverse fetal outcomes at birth, including LGA, small for gestational age (SGA), preterm birth, neonatal mortality, hypoglycemia, hyperglycemia, and MCAs.

\section{METHODS}

Cohort

This was a register-based cohort study in Finland, including children with maternal exposure to metformin or insulin regardless of the indication (GDM, pregestational T2DM or PCOS), born after a pregnancy starting in 2004-2016. The cohort of children was identified using the Finnish Medical Birth Register, which holds information on all births in Finland, including date of birth and gestational age (GA).$^{25}$ The date of start of pregnancy was calculated by subtracting GA (recorded in weeks and days) from the date of delivery to obtain the date of the last menstrual period (LMP). The cohort included singleton children born to women 18-45 years of age at the time of delivery. Exclusion criteria were maternal diagnosis of type 1 diabetes (T1DM), maternal dispensation of systemic glucocorticoids during pregnancy (agents in this drug class are known to interfere with metformin and insulin) and maternal dispensation of antidiabetic medications other than metformin or insulin during pregnancy. To ensure adequate capture of information on exposure and baseline characteristics, children born to women not registered in Finland throughout the entire duration of pregnancy were also excluded. Definitions of all exclusion criteria are provided in online supplemental table S1. Information on emigration and death for the children in the cohort was obtained from the Finnish Population Register Centre, which holds census information for all persons living in Finland.

\section{Exposure}

Information on maternal exposure to the study drugs was obtained from the Finnish Prescription Register, ${ }^{26}$ which contains information on all reimbursed drugs dispensed at all community pharmacies in Finland, including date of dispensation and Anatomical Therapeutic Chemical code (codes for study drugs in online supplemental table S2). Maternal exposure to metformin or insulin was defined as a dispensed prescription in the time period from the LMP until the date of delivery. The children in the cohort were classified into three exposure groups, based on maternal exposure captured via dispensed prescriptions: metformin, insulin, and combination treatment (both metformin and insulin, sequentially or concomitant).

\section{Outcomes}

The primary long-term outcomes were childhood obesity, hypoglycemia, hyperglycemia, hypertension, diabetes (T1DM and T2DM), PCOS (analyzed among girls only), and challenges in MSD. Children were followed from the age of 1 week until the date of the first occurring event of death, emigration, or end of study period (31 December 2016). Cases of the primary outcomes during the follow-up period were ascertained based on diagnosis codes from the Care Register for Health Care (HILMO) and the Register of Primary Health Care Visits (AvoHILMO),${ }^{2728}$ which hold information on contacts in secondary (hospitals) and primary (healthcare centers with general practitioners) healthcare services, respectively. Both registers include information on date of contact (visit or admission) and all diagnoses made (classified according to International Statistical Classification 
of Diseases and Related Health Problems, Tenth Revision codes (ICD-10) in HILMO and ICD-10 and the International Classification of Primary Care, second revision in AvoHILMO).

The secondary outcomes were adverse fetal outcomes at birth: LGA, SGA, preterm birth, neonatal mortality, neonatal hypoglycemia and hyperglycemia and MCAs. Cases of LGA (birth weight two SD above the gestational age-specific and sex-specific reference mean in Finland), SGA (birth weight two SD below the gestational agespecific and sex-specific reference mean), preterm birth (delivery before 37 gestational weeks), and neonatal mortality (death during the first week of life) were identified from the Medical Birth Register. Cases of neonatal hypoglycemia and hyperglycemia were identified using diagnosis codes from HILMO and AvoHILMO and, additionally, plasma glucose values recorded in regional laboratory databases from primary and secondary care; the time point for assessment was up to 28 days after birth. Major congenital malformations were identified using diagnosis codes from the Register of Congenital Malformations and classified according to the EUROCAT classification; ${ }^{29}{ }^{30}$ the time for assessment was up to 1 year of age.

Full definitions of all primary and secondary outcomes are provided in online supplemental table S3.

\section{Confounding control}

Inverse probability of treatment weighting (IPTW) with stabilized weights was used to control for confounding. ${ }^{31}$ The propensity score (PS) estimation (using logistic regression) and weighting was conducted separately for the pairwise comparisons of metformin versus insulin and combination treatment versus insulin. The predictors of the PS models were a broad range of covariates, including demographic factors, comorbidities before and during pregnancy, lifestyle factors and gestational week of GDM diagnosis. Other covariates included were region of residence for the child and calendar year of delivery. The covariates included in the PS estimation are listed in table 1 (covariate definitions in online supplemental table S4). To avoid violations of the positivity assumption, children with an estimated PS outside the overlapping area of the PS distribution in the respective two exposure groups (ie, PS higher than the highest and lower than lowest in the other exposure group) were excluded. ${ }^{31}$ The distributions of the stabilized weights were investigated to identify influential observations; large weights were truncated to a maximum of 10 . Covariate balance after weighting was assessed using the standardized mean difference. A standardized mean difference less than 0.10 was assumed to indicate that the covariate was well balanced. Covariates that were not balanced after weighting were included as separate independent variables in the outcome models.

\section{Statistical analysis}

Incidence rates (IRs) with 95\% CIs were estimated according to age period (description in online supplemental methods 1). Different analytic setups were used to calculate IRs for outcomes considered permanent (diabetes, PCOS, and challenges in MSD) and temporary (obesity, hypoglycemia, hyperglycemia, and hypertension).

The primary outcomes were analyzed using proportional hazards regression to estimate HRs with 95\% CIs. For the analyses of the permanent outcomes, the children in the cohort were followed to the date of the first occurrence of an outcome event. For the temporary outcomes, multiple events per child were allowed. Temporal clustering of events in the same child was taken into account using a time-dependent covariate, indicating whether an event had occurred in the previous 365 days. Additionally, the variance of the outcome models was estimated using a robust estimator. The timescale for all analyses was days since birth. The analyses of the secondary outcomes were conducted using logistic regression to estimate ORs with $95 \%$ CIs. In addition, the mean difference in birth weight associated with maternal exposure to metformin and combination treatment, compared with insulin, was estimated using linear regression. Both unadjusted and IPTW-weighted analyses were conducted.

Two sensitivity analyses for the primary outcomes were conducted. First, the study cohort was restricted to children with maternal GDM. While indications for maternal exposure (ie, GDM, pregestational T2DM, and PCOS) were accounted for in the IPTW weighting, this sensitivity analysis was designed to assess the consistency of results across different indications (description of the subcohort for maternal GDM is provided online supplemental methods 2). Second, the analyses of the primary outcomes were repeated among those with at least two dispensed maternal prescriptions for metformin or insulin at different dates from LMP to birth (as women who refilled prescriptions might be more likely to have used the dispensed medications).

All analyses were conducted using R V.3.5.0.

The study methods were planned prior to conduct and are described in full in the study protocol registered in the European Union PAS Register (EUPAS number 19686).$^{32}$ Of note, the protocol describes two study periods, 1996-2016 and 2004-2016, which was originally intended. However, given the observation of very limited maternal pregnancy exposure to metformin in Finland before 2004, results are presented only for 2004-2016. A description of the numbers exposed in each study period is provided in online supplemental figure $\mathrm{S} 1$.

\section{RESULTS}

A total of 10129 children with maternal exposure to metformin, insulin or both fulfilled the criteria for inclusion. Among these, 3967 were exposed to metformin, 889 were exposed to combination treatment and 5273 were exposed to insulin. For the IPTW-weighted analyses, 296 and 55 exposed to metformin and insulin, respectively, were excluded from the analyses of metformin versus insulin due to non-overlapping PSs; 7 and 21 exposed 


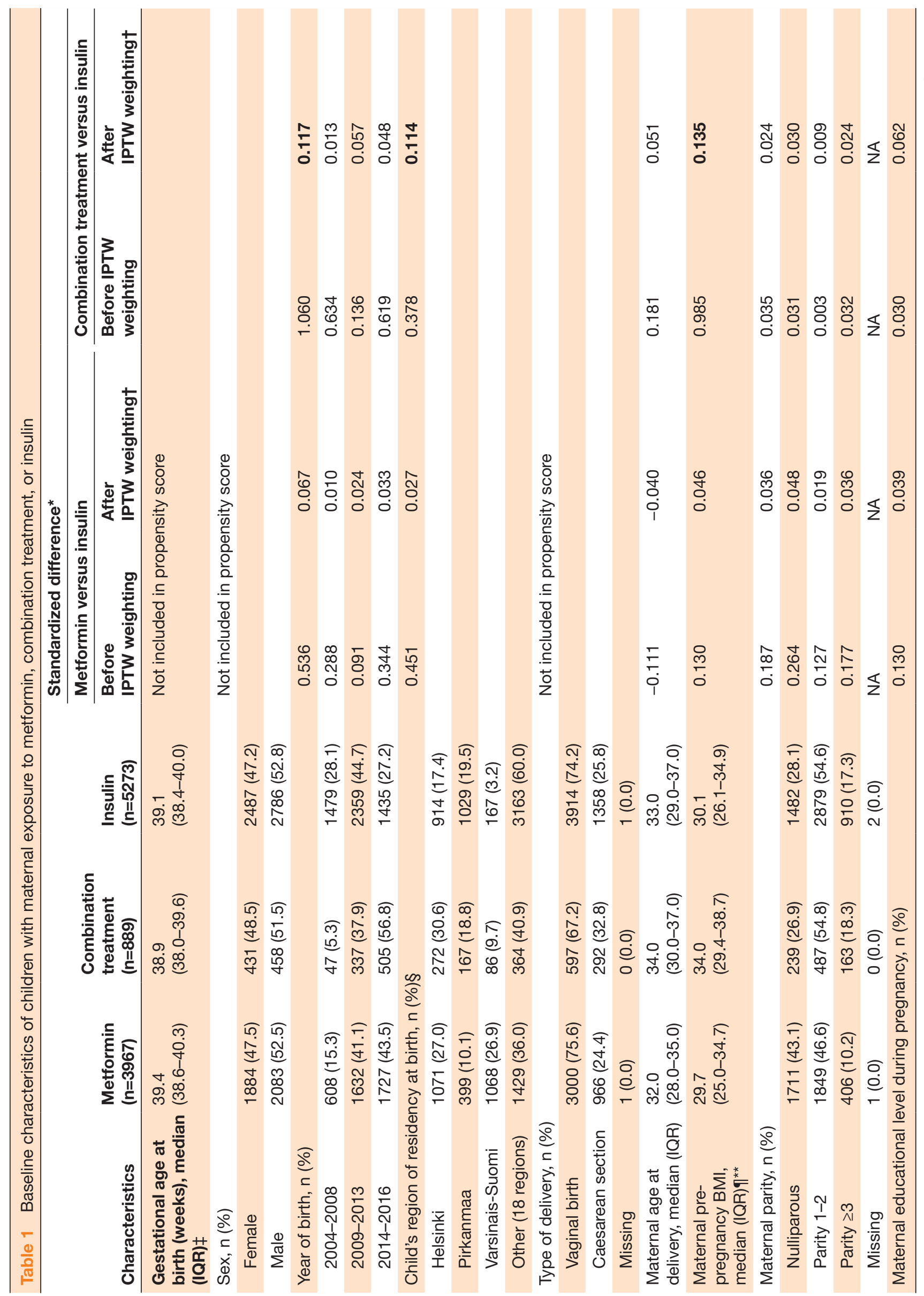




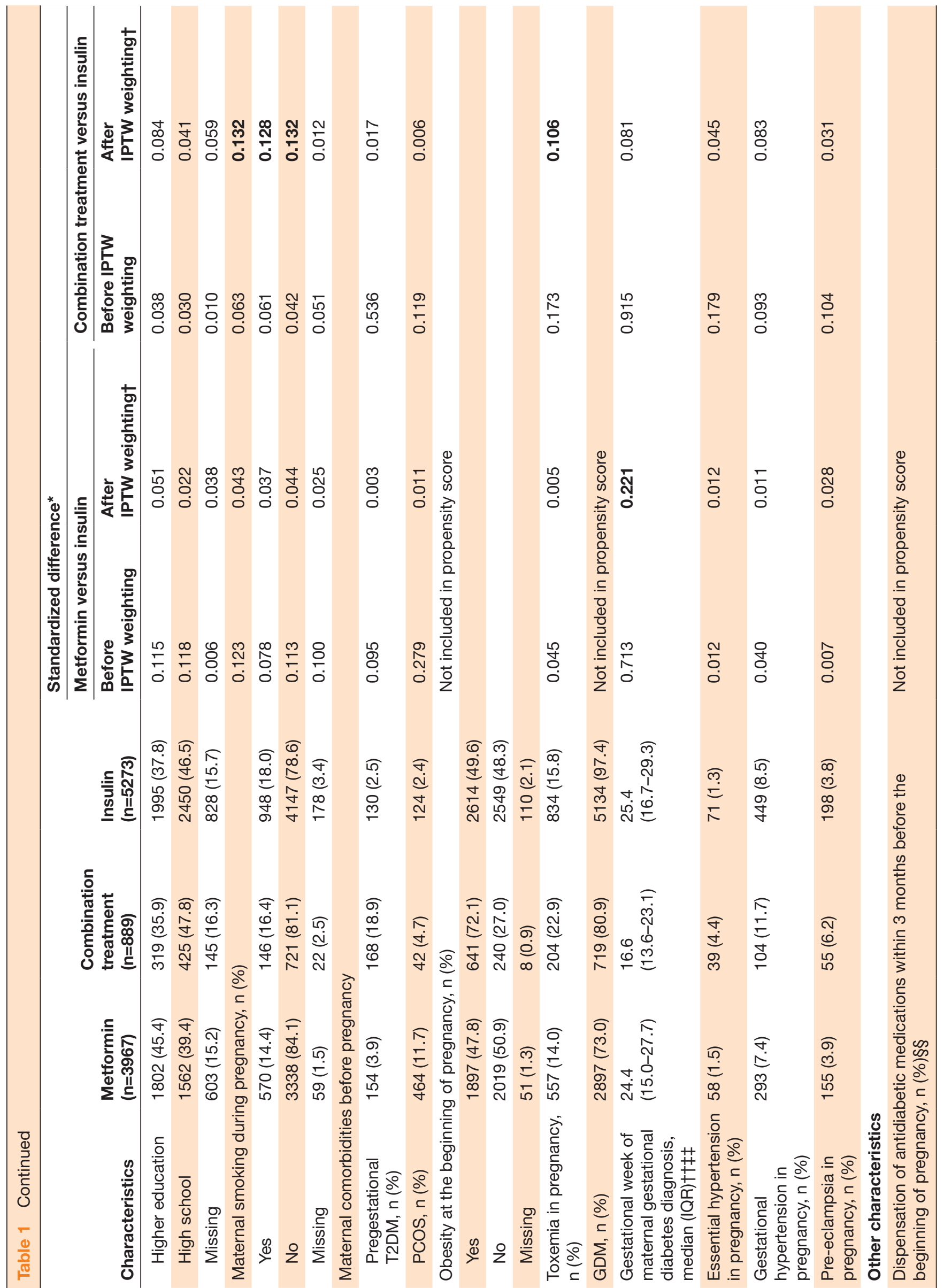



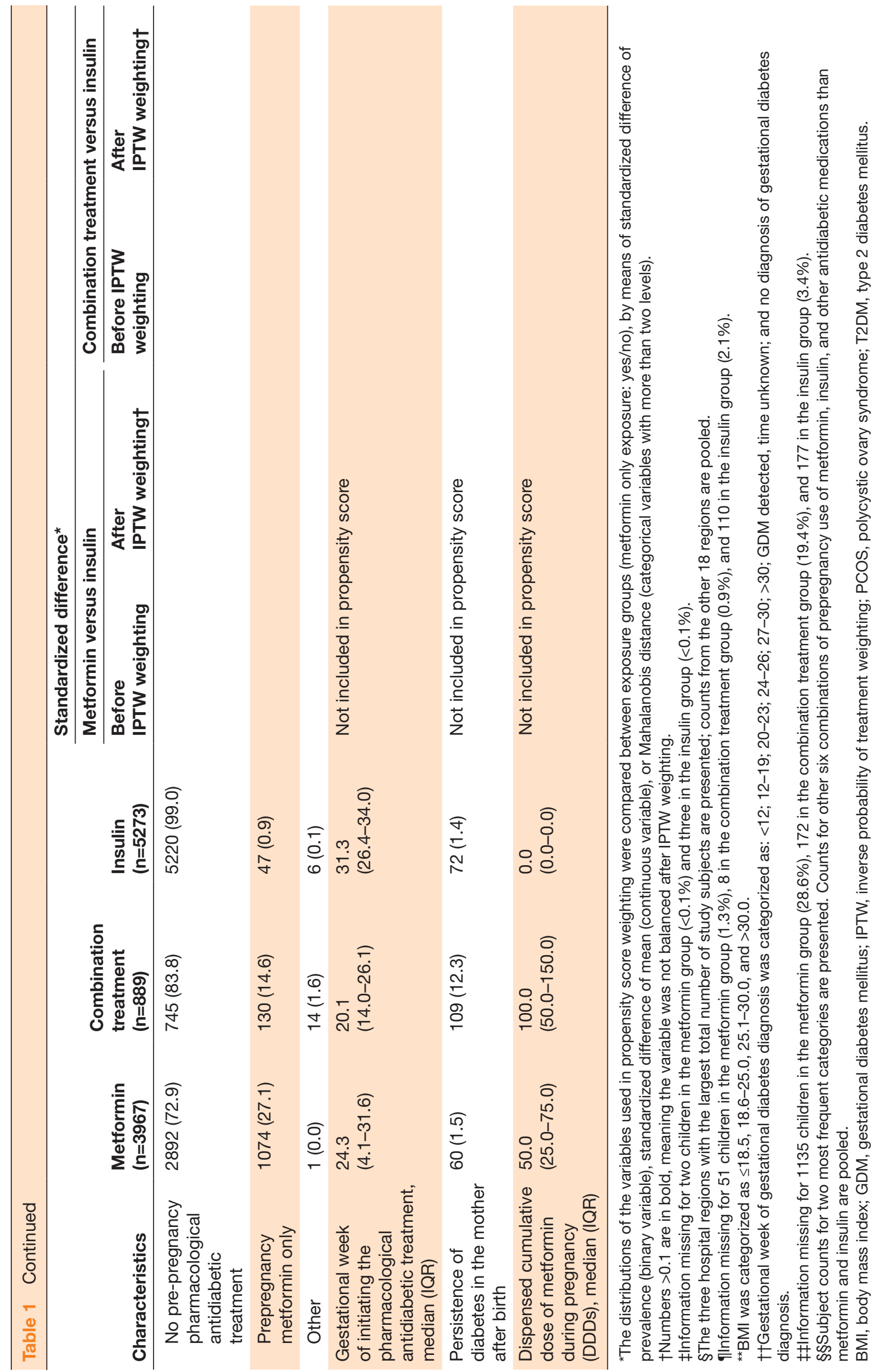
to combination treatment and insulin, respectively, were excluded from the analyses of combination treatment versus insulin.

Baseline characteristics for the children included are shown in table 1 . After weighting, children exposed to metformin and insulin were balanced on all measured characteristics, except week of diagnosis for maternal GDM. Comparing children exposed to combination treatment with those exposed to insulin, after weighting, the exposure groups were balanced on all characteristics, except child's year of birth and region of residence and the maternal characteristics toxemia in pregnancy, smoking, and body mass index.

\section{Primary outcomes}

The median time of follow-up among the children in the cohort was 3.5 years (IQR 1.6-6.4) for those exposed to metformin, 2.4 years (IQR 1.1-4.4) for those exposed to combination treatment, and 5.5 years (IQR 2.8-8.4) for those exposed to insulin.

IRs (per 1000 person-years) for the primary outcomes are presented in table 2. For obesity, the incidence appeared to increase with age; the highest IRs were observed for combination treatment (IR 40.34; 95\% CI 19.23 to 84.61 in the age group $6-8$ years). The large majority of hypoglycemia events were observed in the age group 1 week-2 years; the highest IR was observed in the combination cohort (IR 20.71; 95\% CI 14.94 to 28.72), whereas the IRs in the metformin and in the insulin cohorts were 6.44 (95\% CI 4.98 to 8.33 ) and 4.49 (95\% CI 3.49 to 5.77), respectively. For hyperglycemia, IRs appeared to increase with age, with no marked differences between the exposure groups, however. There were few events of diabetes with no distinct pattern, neither by age nor exposure group. Regarding challenges in MSD, the IR appeared highest in the age periods from 3 to 11 years of age. No distinct pattern according to exposure group could be seen, although, in the youngest age periods ( 1 week-2 years and 3-5 years), the highest IRs were observed for combination treatment (IR 19.99; 95\% CI 14.35 to 27.84 in age group 1 week-2 years and IR 75.42; 95\% CI 56.31 to 101.01 in age group 3-5 years). No events of hypertension or PCOS were observed in the metformin or combination treatment groups.

The results for the primary long-term outcomes are shown in table 3. In weighted analyses, exposure to metformin was not associated with increased risk of obesity (IPTW-weighted HR (wHR) 1.14; 95\% CI 0.83 to 1.55 ), hypoglycemia (wHR 1.00 ; $95 \%$ CI 0.61 to 1.64 ), hyperglycemia (wHR 1.23 ; $95 \%$ CI 0.63 to 2.42 ), diabetes (wHR 1.19; 95\% CI 0.51 to 2.82), or challenges in MSD (wHR 1.09; 95\% CI 0.93 to 1.27). Combination treatment was not associated with increased risk of obesity (wHR 1.09; $95 \%$ CI 0.76 to 1.58), hypoglycemia (wHR 1.14; 95\% CI 0.71 to 1.83 ), hyperglycemia (wHR $0.22 ; 95 \%$ CI 0.05 to 1.01 ), diabetes (wHR $0.14 ; 95 \%$ CI 0.02 to 1.15 ), or challenges in MSD (wHR 1.11; 95\% CI 0.77 to 1.59 ).
Given the absence of events, the risk of hypertension and PCOS could not be estimated.

\section{Sensitivity analyses of the primary outcomes}

The analyses of children with maternal GDM included 2361 children exposed to metformin, 577 exposed to combination treatment, and 4865 exposed to insulin (baseline characteristics in online supplemental table S5). For children exposed to metformin, the results were similar to the main analysis; no significant increased risk of any of the primary outcomes was observed. Exposure to combination treatment was associated with a significantly increased risk of neonatal hypoglycemia (wHR 4.98; $95 \%$ CI 1.28 to 19.35$)$ but not with any other outcome (online supplemental table S6).

In the sensitivity analysis requiring at least two dispensed prescriptions of metformin or insulin for inclusion, results were similar to the main analyses for all primary outcomes, except for a significantly increased risk of challenges in MSD associated with combination treatment (wHR 2.09; 95\% CI 1.21 to 3.61; online supplemental table S7).

\section{Secondary outcomes}

The results of the analyses of the secondary outcomes are shown in table 4. After IPTW weighting, exposure to metformin was associated with significantly lower mean birth weight, compared with insulin (weighted mean difference (wMD) -38.5 g; 95\% CI -60.8 to -16.1), in line with a significantly increased risk of SGA (IPTW-weighted OR (wOR) $1.65 ; 95 \%$ CI 1.16 to 2.34 ). No significantly increased risk associated with metformin for any other secondary outcome was observed in IPTW-weighted analyses. In the analyses of combination treatment, a significantly increased risk was observed for LGA (wOR 1.58; $95 \%$ CI 1.22 to 2.05 ), preterm birth (wOR $1.46 ; 95 \%$ CI 1.10 to 1.95 ), and neonatal hypoglycemia (wOR 1.29; $95 \%$ CI 1.09 to 1.53 ), but not for any other secondary outcome. No significant difference in mean birth weight for combination treatment, compared with insulin, was observed (wMD -9.7 g; 95\% CI -47.6 to 28.2 ).

\section{DISCUSSION}

This large register-based cohort study found that maternal exposure to metformin and combination treatment of metformin and insulin was not associated with long-term increased risk of obesity, hypoglycemia, hyperglycemia, diabetes, or challenges in MSD, compared with insulin. The results were generally consistent in sensitivity analyses restricted to children with maternal GDM. The analyses of adverse outcomes at birth showed significantly lower birth weight and significantly increased risk of SGA associated with exposure to metformin, compared with insulin; combination treatment was associated with increased risk of LGA, preterm birth, and hypoglycemia.

In general, results from this study follow the trend seen in other studies, namely that the obesity risk increases with age of the children. At the age of 3-5 years, the incidence 


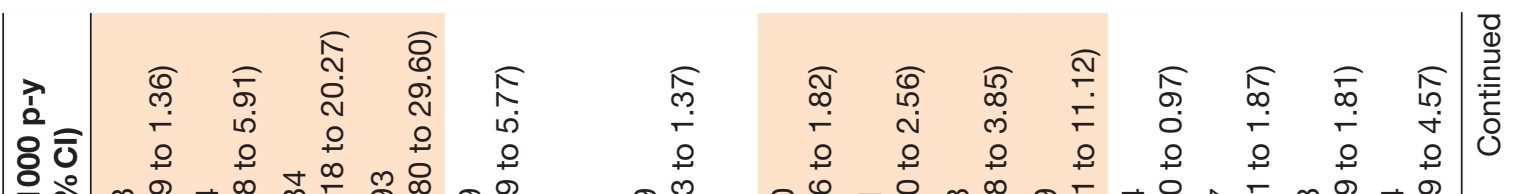
至

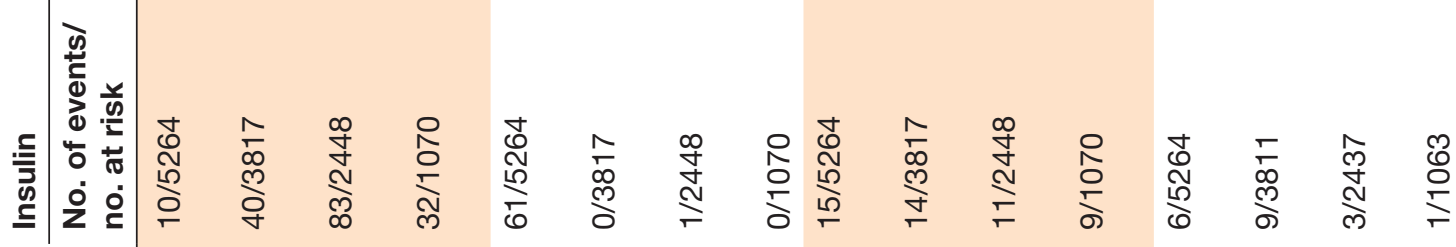

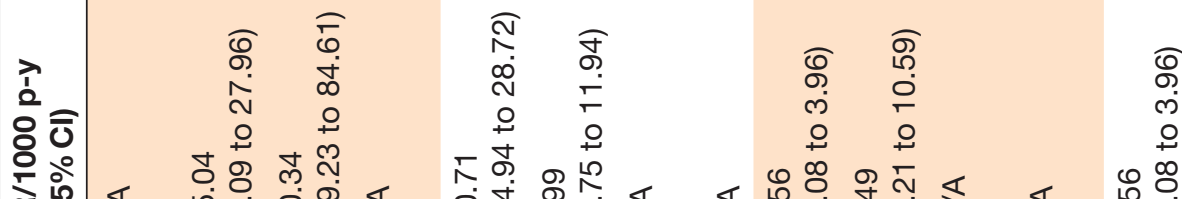

ம்

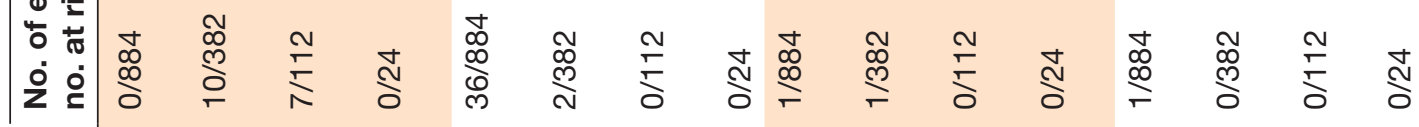

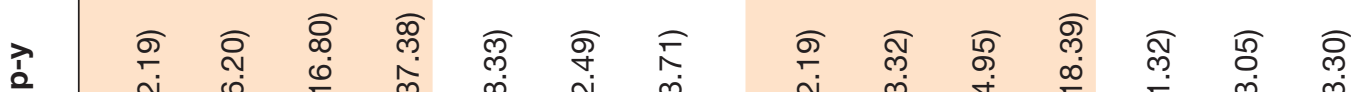

ठำ 商
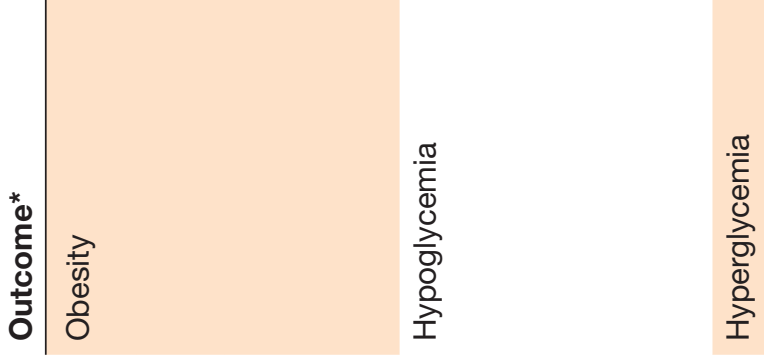


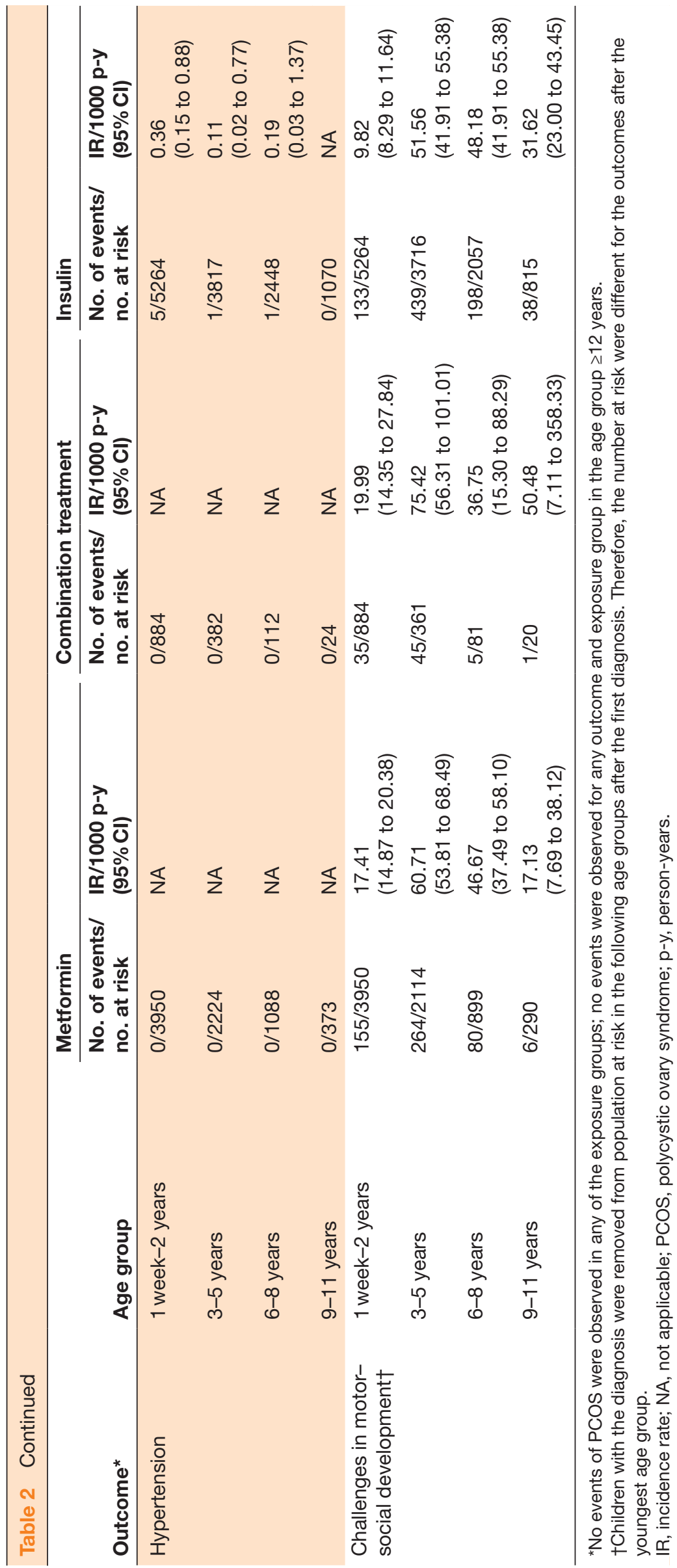


Table 3 Risk of the primary long-term outcomes by exposure group

\begin{tabular}{|c|c|c|c|c|}
\hline \multirow[b]{2}{*}{ Outcome* } & \multicolumn{2}{|c|}{ Unadjusted HR (95\% Cl)† } & \multicolumn{2}{|c|}{ IPTW-weighted HR (95\% CI)‡ } \\
\hline & $\begin{array}{l}\text { Metformin versus } \\
\text { insulin }\end{array}$ & $\begin{array}{l}\text { Combination treatment } \\
\text { versus insulin }\end{array}$ & $\begin{array}{l}\text { Metformin versus } \\
\text { insulin }\end{array}$ & $\begin{array}{l}\text { Combination } \\
\text { treatment } \\
\text { versus insulin }\end{array}$ \\
\hline Obesity & $\begin{array}{l}1.15 \\
(0.82 \text { to } 1.60)\end{array}$ & $\begin{array}{l}1.35 \\
(1.02 \text { to } 1.78)\end{array}$ & $\begin{array}{l}1.14 \\
(0.83 \text { to } 1.55)\end{array}$ & $\begin{array}{l}1.09 \\
(0.76 \text { to } 1.58)\end{array}$ \\
\hline Hypoglycemia & $\begin{array}{l}0.80 \\
(0.49 \text { to } 1.30)\end{array}$ & $\begin{array}{l}1.11 \\
(0.65 \text { to } 1.89)\end{array}$ & $\begin{array}{l}1.00 \\
(0.61 \text { to } 1.64)\end{array}$ & $\begin{array}{l}1.14 \\
(0.71 \text { to } 1.83)\end{array}$ \\
\hline Hyperglycemia & $\begin{array}{l}1.00 \\
(0.61 \text { to } 1.64)\end{array}$ & $\begin{array}{l}0.42 \\
(0.14 \text { to } 1.25)\end{array}$ & $\begin{array}{l}1.23 \\
(0.63 \text { to } 2.42)\end{array}$ & $\begin{array}{l}0.22 \\
(0.05 \text { to } 1.01)\end{array}$ \\
\hline Diabetes mellitus & $\begin{array}{l}1.32 \\
(0.65 \text { to } 2.67)\end{array}$ & $\begin{array}{l}0.70 \\
(0.09 \text { to } 5.21)\end{array}$ & $\begin{array}{l}1.19 \\
(0.51 \text { to } 2.82)\end{array}$ & $\begin{array}{l}0.14 \\
(0.02 \text { to } 1.15)\end{array}$ \\
\hline $\begin{array}{l}\text { Challenges in motor-social } \\
\text { development }\end{array}$ & $\begin{array}{l}1.25 \\
(1.11 \text { to } 1.40)\end{array}$ & $\begin{array}{l}1.58 \\
(1.26 \text { to } 1.98)\end{array}$ & $\begin{array}{l}1.09 \\
(0.93 \text { to } 1.27)\end{array}$ & $\begin{array}{l}1.11 \\
(0.77 \text { to } 1.59)\end{array}$ \\
\hline
\end{tabular}

IPTW-weighted HR (95\% Cl)‡

*No events of the long-term primary outcomes hypertension and PCOS were observed in the metformin or combination treatment groups. †Metformin and combination treatment were compared with insulin. In the unadjusted comparison, 3967 children were exposed to metformin; 889 children were exposed to combination treatment; and 5273 were exposed to insulin.

$\ddagger$ Metformin and combination treatment were analyzed separately, in pairwise comparisons with insulin (reference in all analyses). Analyses were conducted in the main cohort after trimming of children outside the overlapping range of the propensity score. In the IPTW analyses of metformin versus insulin, 3671 children exposed to metformin and 5218 exposed to insulin included; in the analyses of combination treatment versus insulin, 882 children exposed to combination treatment and 5252 exposed to insulin included.

IPTW, inverse probability of treatment weighting; PCOS, polycystic ovary syndrome.

of obesity after metformin was comparable with that of insulin, in line with two GDM follow-ups from Finland and New Zealand, which found no increased risk of obesity associated with maternal exposure to metformin at 18 months and 4 years of age, respectively. ${ }^{15} 18$ In the age group $6-8$ years, the obesity incidence was still more than twice as high in the combination treatment group compared with the metformin and insulin groups, but the IPTW-related HR was not significantly increased, suggesting a strong influence by confounders. In the long-term outcomes of the Australian GDM study, ${ }^{16}$ no stratification by the use of metformin alone or in combination with insulin was made, but still the results differ. Two Norwegian RCT follow-up studies in children with maternal PCOS also found increased risk of obesity at a follow-up of 4 and up to 10 years of age, compared with placebo. ${ }^{2021}$ The key differences in study population, that is, metformin used for GDM or T2DM or PCOS in this study versus single indications in the prospective studies and treatment comparator, that is, metformin with/ without supportive insulin versus insulin and placebo, respectively, make this comparison difficult. Nevertheless, this study represents the largest investigation of the long-term association between obesity and maternal exposure to metformin at the age of 9-12 years to date, and in this age group, exposure to metformin and insulin had comparable obesity IRs.

To our knowledge, this is the first study to investigate the association between exposure to metformin and risk of hypoglycemia or hyperglycemia beyond the age of 1 week, aiming to capture incidents of prolonged neonatal hyperinsulinemia or permanent alterations to metabolism. A small number of previous studies have investigated glucose levels among children with maternal exposure to metformin, observing no significant increase compared with insulin or placebo. ${ }^{162} 33$ In this study, neither metformin nor combination treatment was associated with increased risk of hypoglycemia or hyperglycemia. Notably, combination treatment was associated with lower risk of hyperglycemia, although not significantly and based on few events. In the analyses among children with maternal GDM, combination treatment was associated with increased risk of hypoglycemia within the first 2 years, probably due to the prolonged postnatal hyperinsulinism, as mothers prescribed both insulin and metformin likely represent those with the most severe gestational diabetes. ${ }^{34} 35$

The previous evidence regarding long-term risk of hypertension, diabetes, and PCOS associated with maternal exposure to metformin is scarce; to our knowledge, no previous investigations have been published. In this study, no cases of hypertension or PCOS were identified, which prevents conclusions regarding these outcomes being drawn. Indeed, PCOS is generally not diagnosed before late adolescence, which may explain the absence of events for this outcome. ${ }^{36}$ For diabetes, exposure to metformin was not associated with increased risk; combination treatment was associated with nonsignificantly lower risk, although based on few events.

The finding of no increased risk in the main analyses of long-term challenges in MSD aligns with previous reports assessing developmental outcomes with maternal exposure to metformin. ${ }^{151823}$ However, increased risk associated with combination treatment was observed in the sensitivity analysis requiring two prescriptions. Notably, the incidence of challenges in MSD showed a clear 


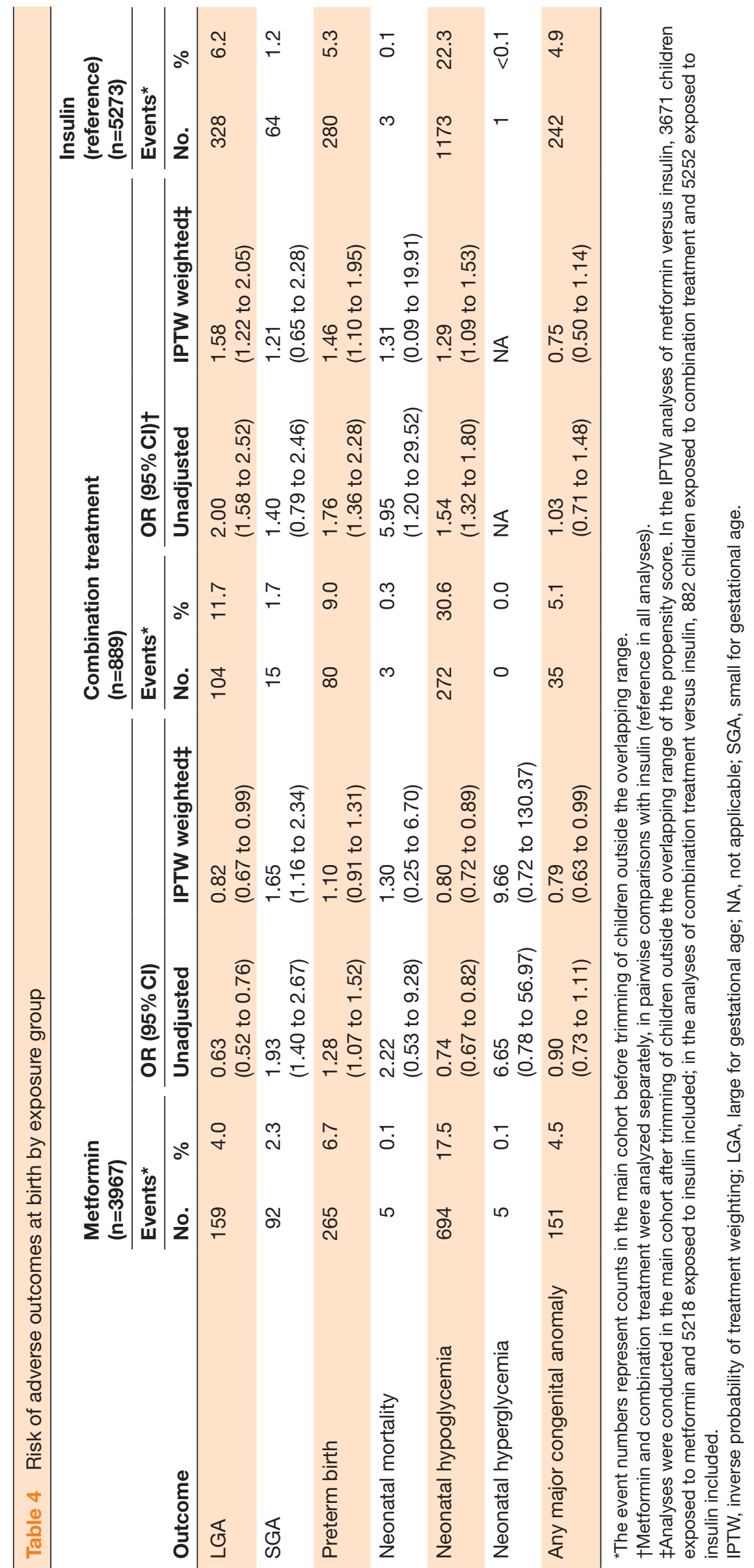


increase over calendar time (data not shown), which may be explained by the availability of data from primary care only from 2011. Coupled with imbalances in year of birth between the combination treatment and insulin groups (standardized difference after weighting $=0.117$ ), this could potentially explain the observed risk increase. The current study expands on the available evidence by providing analyses at a follow-up of up to 12 years, compared with a maximum 4-year follow-up in previous data. ${ }^{18}$

In the analyses of SGA, maternal exposure to metformin was associated with increased risk versus insulin. While a significant association between metformin alone and SGA has not been previously reported, a meta-analysis of RCTs found a non-significant tendency toward increased risk, compared with insulin. ${ }^{9}$ Several potential pathways through which metformin may influence risk of SGA have been suggested, including reduced maternal food intake, inhibition of the mammalian target of rapamycin (mTOR), and inhibition of folate-related pathways. ${ }^{24}$ As metformin crosses the placenta, ${ }^{5-7}$ direct fetal effects, for example, affecting the fetal metabolic milieu and cell metabolism, are also plausible. ${ }^{124}$ Notably, a recent RCT found an almost twofold significantly increased risk of SGA associated with combination treatment (metformin and insulin), compared with insulin alone. ${ }^{24}$ Possible correlations between SGA birth and maternal variables after exposure to metformin may be subject to a follow-up analysis.

Previous evidence regarding metformin and LGA and macrosomia is conflicting; while most reports have not found an association, ${ }^{37-39}$ some studies and meta-analyses have reported lower risk compared with insulin. ${ }^{9}$ 40-42 Furthermore, a recent RCT found that maternal combination treatment (metformin and insulin) was associated with lower risk of being extremely LGA, compared with insulin alone. ${ }^{24}$ Given that increased risk of LGA was only observed for combination treatment (ie, the exposure group including those with the likely most severe types of diabetes and the highest maternal BMI) in this study and, also, given the known association between glucose control and birth weight, confounding by disease severity appears a probable explanation.

In the analyses of preterm birth and neonatal hypoglycemia, an increased risk was observed only for combination treatment. Although previous data on preterm birth is conflicting, ${ }^{9} 10373843$ residual or unmeasured confounding appears as a possible explanation. Specifically, several risk factors for preterm birth (including maternal BMI, toxemia, and smoking) remained unbalanced between the combination treatment and insulin groups after weighting and may not have been fully accounted for.

With regard to MCAs, no increased risk was observed, neither for metformin alone nor combination treatment. This is consistent with previous reports that found no increased risk of major congenital malformations associated with metformin, compared with, respectively, insulin, non-exposure to metformin, and non-exposure to any diabetic medication. ${ }^{11-1344-46}$

This study had several strengths. First, the use of the comprehensive national Finnish health registers allowed for long-term follow-up, extending up to 12 years of age. Second, the nationwide coverage of data likely provided high representativeness and generalizability. Third, information on drug exposure was ascertained from a national prescription register, likely providing high completeness and precision regarding timing of use. Fourth, the use of IPTW methods based on PSs including a broad range of maternal characteristics reduced the potential for confounding by baseline characteristics.

There were also limitations. First, non-use of dispensed drugs would lead to exposure misclassification; given that metformin is known to more commonly be discontinued than insulin (eg, due to gastrointestinal side effects), this could potentially obscure a true association. However, in sensitivity analyses requiring at least two dispensed prescriptions for inclusion, the results were similar to those in the main analysis. For the combination treatment group, it was not possible to determine if metformin and insulin were used sequentially or concomitantly. Although the content of the Finnish national health registers is known to be of satisfactory to very good quality, ${ }^{47}$ outcome misclassification remains possible. In particular, the ascertainment of the long-term outcomes relied on diagnoses that have not been formally validated for sensitivity or specificity. It is possible that sensitivity may have been limited for some outcomes, including obesity and less severe events of hypoglycemia. If sensitivity and misclassification was non-differential between exposure groups, this would likely bias results toward no effect. Also, the study population consisted of live births; thus, MCAs that led to elective termination of pregnancy were not included. In addition, the metformin group included children with maternal GDM, T2DM, and PCOS, whereas maternal indications for insulin include GDM and T2DM alone. While indications for treatment were accounted for in the IPTW, any remaining unmeasured differences in baseline risk between the groups would lead to confounding. However, in sensitivity analysis restricted to children with maternal GDM, the results were similar to the main analysis. While the study allowed for 12-year follow-up, the median follow-up time ( 3.5 years) was relatively short, thus limiting the sample size and precision at the older age periods. Still the number of children in the 9-11 year period is larger than in any other report we are aware of. Furthermore, although it has an influence on long-term outcomes, notably childhood obesity, breast feeding was not accounted for in the study as information on breast feeding is not available in Finnish data sources. However, it is unlikely that breast feeding would vary differentially across exposure groups. ${ }^{48}$ Also, metformin passes into the maternal milk with very low to neglectable concentrations and breastfed versus formula-fed infants did not show a difference after 6 months. ${ }^{49}$ Therefore, it is unlikely that adjustment for breast feeding would 
considerably change the findings. Finally, maternal disease severity (eg, level of glucose control) and individual risk factors (eg, gestational weight gain) could not be accounted for in the analysis, although being known potential confounders.

Although a longer median follow-up time could bring better confidence to its findings, this study found no increased long-term risk of obesity, hypoglycemia, hyperglycemia, diabetes, or challenges in MSD associated with in utero exposure to metformin (alone or in combination with insulin), compared with insulin alone. The observed increased risk of SGA associated with metformin alone versus insulin may warrant caution for use in pregnancies with risk of foetal undernutrition. The associations between combination treatment and increased risk of LGA, preterm birth, and hypoglycemia may be explained by confounding.

Acknowledgements The study team would like to thank additional helping hands on this publication from both Merck and IQVIA, namely Ulrike Gottwald-Hostalek, Yorki Tayrouz, Patrice Verpillat, Mounir Ould Setti and Urmeli Katus. We further acknowledge Saara Hetemäki as the project manager and data access lead of this research study; Rosa Juuti for her contribution on the conceptualization of the study, the study protocol and the study permits; Anna Glans Lundin for her contribution on the study protocol; and Juha Mehtälä for his contribution in the study design and planning of the statistical analyses. The study team warmly thanks lan Campbell for his dedication and wisdom regarding metformin for over 40 years, which has changed much to the better, including this study.

Collaborators Members of the CLUE Study Group are Minna Vehkala (Global Database Studies, IQVIA), Robyn Thorén (Global Database Studies, IQVIA), Henrik Svanström (Global Database Studies, IQVIA).

Contributors KMGB developed the study idea, backbone and synopsis as the outcome of a cumulative assessment on the benefit/risk profile of metformin during pregnancy in 2016, in her role as Global Medical representative of the metformin originator Merck KGaA, Darmstadt, Germany . KMGB represented the company, which was the study sponsor, acts as guarantor and significantly contributed to the manuscript. JuS, CF and EB (all Merck KGaA, Darmstadt, Germany) contributed to finetuning the study concept, protocol development, data analysis and manuscript. The study was conducted by IQVIA, contracted by Merck KGaA, Darmstadt, Germany. LM-P and MV provided independent medical expertise to the study and manuscript.

Funding The authors disclosed receipt of the following financial support for the research, authorship, and/or publication of this article: this study was funded by Merck KGaA, Darmstadt, Germany (CrossRef Funder ID: 10.13039/100009945).

Competing interests KMGB, CF, EB, and JuS are employees of Merck Healthcare KGaA, Darmstadt, Germany, the metformin originator and sponsor of this study, and participated in the authoring/revision of the manuscript. IQVIA performs commissioned pharmacoepidemiological studies, including this one, for several pharmaceutical companies. LS, JaS, MV, RT and KMH are employees of IQVIA, which performs commissioned pharmacoepidemiological studies for several pharmaceutical companies. HS is an employee of IQVIA and has received consulting fees from Celgene, outside the submitted work. PK was at the time of conducting the study an employee of IQVIA.

Patient consent for publication Not applicable.

Ethics approval The Ethics Committee of the Hospital District of Helsinki and Uusimaa, Finland, granted the study a favourable approval. Reference number is HUS/1742/2017. This study was a retrospective analysis, therefore, informed consent by participants was not required.

Provenance and peer review Not commissioned; externally peer reviewed.

Data availability statement All data relevant to the study are included in the article or uploaded as supplementary information. Study documents are available at ENCePP with EU PAS Register Number EUPAS19686.

Supplemental material This content has been supplied by the author(s). It has not been vetted by BMJ Publishing Group Limited (BMJ) and may not have been peer-reviewed. Any opinions or recommendations discussed are solely those of the author(s) and are not endorsed by BMJ. BMJ disclaims all liability and responsibility arising from any reliance placed on the content. Where the content includes any translated material, BMJ does not warrant the accuracy and reliability of the translations (including but not limited to local regulations, clinical guidelines, terminology, drug names and drug dosages), and is not responsible for any error and/or omissions arising from translation and adaptation or otherwise.

Open access This is an open access article distributed in accordance with the Creative Commons Attribution Non Commercial (CC BY-NC 4.0) license, which permits others to distribute, remix, adapt, build upon this work non-commercially, and license their derivative works on different terms, provided the original work is properly cited, appropriate credit is given, any changes made indicated, and the use is non-commercial. See: http://creativecommons.org/licenses/by-nc/4.0/.

\section{ORCID IDs}

Kerstin M G Brand http://orcid.org/0000-0002-8374-4026

Caroline Foch http://orcid.org/0000-0002-0254-5560

\section{REFERENCES}

1 American Diabetes Association. 9. Pharmacologic Approaches to Glycemic Treatment: Standards of Medical Care in Diabetes-2019. Diabetes Care 2019:42:S90-102.

2 Buse JB, Wexler DJ, Tsapas A, et al. 2019 update to: management of hyperglycemia in type 2 diabetes, 2018. a consensus report by the American diabetes association (ADA) and the European association for the study of diabetes (EASD). Diabetes Care 2020;43:487-93.

3 Cesta CE, Cohen JM, Pazzagli L, et al. Antidiabetic medication use during pregnancy: an international utilization study. BMJ Open Diabetes Res Care 2019;7:e000759.

4 Finnish Medical Society Duodecim. Raskausdiabetes: Käypä hoito -suositus [in Finnish], 2013. Available: http://www.kaypahoito.fi/web/

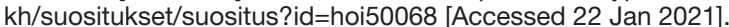

5 Pollex EK, Feig DS, Lubetsky A, et al. Insulin glargine safety in pregnancy: a transplacental transfer study. Diabetes Care 2010;33:29-33.

6 Suffecool K, Rosenn B, Niederkofler EE, et al. Insulin detemir does not cross the human placenta. Diabetes Care 2015;38:e20-1.

7 Boskovic R, Feig DS, Derewlany L, et al. Transfer of insulin lispro across the human placenta: in vitro perfusion studies. Diabetes Care 2003:26:1390-4.

8 de Oliveira Baraldi C, Lanchote VL, de Jesus Antunes N, et al. Metformin pharmacokinetics in nondiabetic pregnant women with polycystic ovary syndrome. Eur J Clin Pharmacol 2011;67:1027-33.

9 Butalia S, Gutierrez L, Lodha A, et al. Short- and long-term outcomes of metformin compared with insulin alone in pregnancy: a systematic review and meta-analysis. Diabet Med 2017;34:27-36.

10 Rowan JA, Hague WM, Gao W, et al. Metformin versus insulin for the treatment of gestational diabetes. N Engl J Med 2008;358:2003-15.

11 Diav-Citrin O, Steinmetz-Shoob S, Shechtman S, et al. In-utero exposure to metformin for type 2 diabetes or polycystic ovary syndrome: a prospective comparative observational study. Reprod Toxicol 2018;80:85-91.

12 Panchaud A, Rousson V, Vial T, et al. Pregnancy outcomes in women on metformin for diabetes or other indications among those seeking teratology information services. Br J Clin Pharmacol 2018;84:568-78.

13 Scherneck S, Schlinke N, Beck E, et al. Pregnancy outcome after first-trimester exposure to metformin: a prospective cohort study. Reprod Toxicol 2018;81:79-83.

14 ljäs $\mathrm{H}$, Vääräsmäki M, Morin-Papunen L, et al. Metformin should be considered in the treatment of gestational diabetes: a prospective randomised study. BJOG 2011;118:880-5.

15 ljäs $\mathrm{H}$, Vääräsmäki $\mathrm{M}$, Saarela $\mathrm{T}$, et al. A follow-up of a randomised study of metformin and insulin in gestational diabetes mellitus: growth and development of the children at the age of 18 months. BJOG 2015;122:994-1000.

16 Rowan JA, Rush EC, Plank LD, et al. Metformin in gestational diabetes: the offspring follow-up (mig tofu): body composition and metabolic outcomes at 7-9 years of age. BMJ Open Diabetes Res Care 2018;6:e000456

17 Tertti K, Toppari J, Virtanen HE, et al. Metformin treatment does not affect testicular size in offspring born to mothers with gestational diabetes. Rev Diabet Stud 2016;13:59-65.

18 Landi SN, Radke S, Engel SM, et al. Association of long-term child growth and developmental outcomes with metformin vs insulin treatment for gestational diabetes. JAMA Pediatr 2019;173:160-8. 
19 Vanky E, Stridsklev S, Heimstad R, et al. Metformin versus placebo from first trimester to delivery in polycystic ovary syndrome: a randomized, controlled multicenter study. J Clin Endocrinol Metab 2010;95:E448-55.

20 Hanem LGE, Stridsklev S, Júlíusson PB, et al. Metformin use in PCOS pregnancies increases the risk of offspring overweight at 4 years of age: follow-up of two RCTs. J Clin Endocrinol Metab 2018;103:1612-21.

21 Hanem LGE, Salvesen Øyvind, Juliusson PB, et al. Intrauterine metformin exposure and offspring cardiometabolic risk factors (PedMet study): a 5-10 year follow-up of the PregMet randomised controlled trial. Lancet Child Adolesc Health 2019;3:166-74.

22 Panagiotopoulou O, Syngelaki A, Georgiopoulos G, et al. Metformin use in obese mothers is associated with improved cardiovascular profile in the offspring. Am J Obstet Gynecol 2020;223:246.e1-246. e10.

23 Tertti K, Eskola E, Rönnemaa T, et al. Neurodevelopment of twoyear-old children exposed to metformin and insulin in gestational diabetes mellitus. J Dev Behav Pediatr 2015;36:752-7.

24 Feig DS, Donovan LE, Zinman B, et al. Metformin in women with type 2 diabetes in pregnancy (MiTy): a multicentre, international, randomised, placebo-controlled trial. Lancet Diabetes Endocrinol 2020;8:834-44.

25 Finnish Institute for Health and Welfare. Medical birth register: quality description, 2018. Available: https://thl.fi/en/web/thlfi-en/statistics/ information-on-statistics/quality-descriptions/parturients-deliversand-newborns [Accessed 18 Feb 2020].

26 Social Insurance Institution. Finnish Prescription Register: Quality description 2019. (In Finnish: Laatuseloste 2019: Tilasto korvatuista resepteistä) [in Finnish], 2019. Available: http://www.kela.fi/ laatuseloste-2015-tilasto-korvatuista-resepteista\#4 [Accessed 18 Feb 2020].

27 Care Register for Health Care - THL. Finnish Institute for Health and Welfare (THL), Finland. Available: https://thl.fi/en/web/thlfi-en/ statistics/information-on-statistics/register-descriptions/careregister-for-health-care [Accessed 04 Jun 2020].

28 Register of primary health care visits - THL. Finnish Institute for Health and Welfare (THL), Finland. Available: https://thl.fi/en/web/ thlfi-en/statistics/information-on-statistics/register-descriptions/ register-of-primary-health-care-visits [Accessed 04 Jun 2020].

29 Finnish Institute for Health and Welfare. Congenital anomalies: quality description, 2018. Available: http://thl.fi:8080/en/web/thlfi-en/ statistics/information-on-statistics/quality-descriptions/congenitalanomalies [Accessed 31 Jan 2020].

30 EUROCAT. EUROCAT guide 1.4, 2018. Available: https://eu-rdplatform.jrc.ec.europa.eu/eurocat/data-collection/guidelines-fordata-registration [Accessed 30 Jan 2020].

31 Stürmer T, Wyss R, Glynn RJ, et al. Propensity scores for confounder adjustment when assessing the effects of medical interventions using nonexperimental study designs. J Intern Med 2014:275:570-80.

32 Eu PAS register: consequences for life of children with in utero exposure to metformin in Finland - a register-based cohort study (EUPAS19686), 2019. Available: http://www.encepp.eu/encepp/ viewResource.htm?id=31566 [Accessed 22 Oct 2020].
33 Rø TB, Ludvigsen HV, Carlsen SM, et al. Growth, body composition and metabolic profile of 8-year-old children exposed to metformin in utero. Scand J Clin Lab Invest 2012;72:570-5.

34 McGowan JE. Neonatal hypoglycemia. Pediatrics In Review 1999;20:e6-15.

35 Yadav D, Hypoglycemia PN. Hypoglycemia - Causes and Occurrences. Dover, DE, USA: SMGroup, 2016: 1-15.

36 Teede HJ, Misso ML, Costello MF, et al. Recommendations from the International evidence-based guideline for the assessment and management of polycystic ovary syndrome. Hum Reprod 2018;33:1602-18.

37 Tertti K, Ekblad U, Vahlberg T, et al. Comparison of metformin and insulin in the treatment of gestational diabetes: a retrospective, case-control study. Rev Diabet Stud 2008;5:95-101.

38 Syngelaki A, Nicolaides $\mathrm{KH}$, Balani J, et al. Metformin versus placebo in obese pregnant women without diabetes mellitus. N Engl J Med 2016;374:434-43.

39 Mateus J, Ramos LS, Gonzalez K, et al. 890: best gestational diabetes therapy to reduce adverse maternal outcomes. A network meta-analysis. Am J Obstet Gynecol 2020;222:S554-5.

40 Tarry-Adkins JL, Aiken CE, Ozanne SE. Neonatal, infant, and childhood growth following metformin versus insulin treatment for gestational diabetes: a systematic review and meta-analysis. PLOS Med 2019;16:e1002848.

41 Bao L-X, Shi W-T, Han Y-X. Metformin versus insulin for gestational diabetes: a systematic review and meta-analysis. J Matern Fetal Neonatal Med 2021;34:2741-53.

42 Zhen XM, Li X, Chen C. Metformin versus insulin for gestational diabetes: the reporting of ethnicity and a meta-analysis combining English and Chinese literatures. Obes Med 2018;11:48-58.

43 Løvvik TS, Carlsen SM, Salvesen Øyvind, et al. Use of metformin to treat pregnant women with polycystic ovary syndrome (PregMet2): a randomised, double-blind, placebo-controlled trial. Lancet Diabetes Endocrinol 2019:7:256-66.

44 Kjerpeseth L, Cesta C, Engeland A. Risk of major congenital malformations with metformin compared with insulin in pregnancy. EASD 2020 https://www.easd.org/virtualmeeting/home.html\#! resources/risk-of-major-congenital-malformations-with-metformincompared-with-insulin-in-pregnancy-34eb45fc-47e3-499d-b1f7d711c408c2c9

45 Lin S-F, Chang S-H, Kuo C-F, et al. Association of pregnancy outcomes in women with type 2 diabetes treated with metformin versus insulin when becoming pregnant. BMC Pregnancy Childbirth 2020;20:512.

46 Given JE, Loane M, Garne E, et al. Metformin exposure in first trimester of pregnancy and risk of all or specific congenital anomalies: exploratory case-control study. BMJ 2018;361:k2477.

47 Sund R. Quality of the Finnish hospital discharge register: a systematic review. Scand J Public Health 2012;40:505-15.

48 Kole-White MB, Griffin L, Ding JJ, et al. Breastfeeding success among women with gestational diabetes managed by diet only compared with those requiring medications. Breastfeed Med 2021:16:419-23.

49 Glueck CJ, Salehi M, Sieve L, et al. Growth, motor, and social development in breast- and formula-fed infants of metformin-treated women with polycystic ovary syndrome. J Pediatr 2006;148:628-32. 